\title{
Factores asociados al inicio de la lactancia materna en mujeres dominicanas ${ }^{1}$
}

\author{
Leonelo E. Bautista²
}

RESUMEN Las relaciones entre el inicio del amamantamiento y sus determinantes varían en distintas poblaciones, pero su conocimiento es fundamental para orientar los programas de promoción de la lactancia materna. Los datos de la Encuesta Demográfica y de Salud de 1991 de la República Dominicana se usaron para identificar factores asociados con el inicio del amamantamiento en una muestra aleatoria de mujeres en edad fértil. Aproximadamente 93\% de 2714 madres informaron haber iniciado el amamantamiento en su último niño nacido vivo menor de 5 años y no hubo cambios importantes en este porcentaje en los últimos 5 años. Se realizó un análisis de regresión logística mediante el cual se calcularon valores de la razón de posibilidades ( $u$ odds ratio $(\psi)$, en inglés) como medidas de asociación. Las madres que tuvieron alguna enfermedad durante el embarazo $(\psi=2,3)$, las que tuvieron niños con bajo peso al nacer $(\psi=2,9)$, las primíparas $(\psi=1,9)$ y las de nivel de ingresos medio $(\psi=1,6)$ y alto $(\psi=2,1)$ tuvieron un riesgo significativamente mayor de no iniciar el amamantamiento. Estas mujeres deberían constituir grupos prioritarios en los programas de promoción de la lactancia materna.

Los efectos favorables de la lactancia materna en la salud del niño son ampliamente conocidos. Son muchas las pruebas de que la leche materna disminuye la incidencia y reduce la gravedad de las enfermedades infecciosas, principalmente las diarreas agudas $(1,2)$ y las infecciones respiratorias agudas $(3,4)$. Además, la lactancia materna es la forma de alimentación más apropiada para garantizar un crecimiento y desarrollo adecuado durante los primeros 6 meses de vida (5). También genera beneficios indirectos sobre la salud de la madre y el niño por medio de la prolongación de los

\footnotetext{
1 Esta investigación se realizó con financiamiento del Institute for Resources Development (IRD), Macro/International. Próximamente en esta revista se publicará una versión en inglés de este artículo.

2 Universidad Industrial de Santander, Facultad de Salud, Departamento de Salud Pública, Carrera 32, Calles 29 y 30, 3er. piso, Bucaramanga, Colombia.
}

intervalos intergenésicos y la disminución de la fertilidad (6) y el gasto en alimentación y en asistencia médica.

Los efectos beneficiosos de la lactancia materna dependen de su inicio, su duración y la edad de ablactación del niño $(4,7)$. Se sabe que las relaciones entre el inicio de la lactancia al seno y sus determinantes no son iguales en distintos países (8-10). Por lo tanto, el estudio de estas relaciones en poblaciones específicas es de gran importancia para orientar de manera objetiva y efectiva las acciones de promoción y prevenir la disminución en el inicio de la lactancia materna que parece ocurrir en países en desarrollo debido al rápido progreso de la urbanización, la transculturación y la influencia de los grupos sociales más acomodados sobre los más desfavorecidos $(8,11)$.

La información existente sobre lactancia materna en la República Domi- nicana es limitada y las encuestas demográficas y de salud de 1986 y 1991 (12, 13) son probablemente las fuentes de datos más representativas y actualizadas sobre el problema. Sin embargo, tanto estas encuestas como otros estudios (12-15) se limitan a una descripción de las características de la lactancia al seno. En el presente estudio los datos de la Encuesta Demográfica y de Salud de 1991 (ENDESA-91) (13) fueron reanalizados a fin de identificar cuáles características sociodemográficas, de la atención médica, del embarazo y del neonato influyen de forma independiente en el inicio de la lactancia materna.

\section{MATERIAL Y MÉTODOS}

Se realizó un estudio de cohorte utilizando los datos de la ENDESA-91. 
Esta encuesta (13) es parte del Programa de Encuestas Demográficas y de Salud implementado desde 1984 en varios países por el Instituto para el Desarrollo de Recursos y el Consejo de Población y financiado por la Agencia de los Estados Unidos para el Desarrollo Internacional (USAID). La encuesta se realizó a 7142 mujeres dominicanas de 15 a 49 años de edad, integrantes de una muestra seleccionada mediante muestreo probabilístico por conglomerados, multietápico y estratificado. Por medio de entrevista a la madre se recogieron datos retrospectivos sobre el inicio de la lactancia materna en los nacidos vivos en los últimos 5 años (13).

Para el presente estudio solo se consideraron elegibles las parejas madreúltimo niño nacido vivo en las cuales el niño tenía menos de 60 meses de edad en el momento de la encuesta. La selección del último niño se debió a que el período de recordación de los datos de este niño era más corto que los períodos correspondientes a niños nacidos previamente. Además, el uso de la información sobre el último nacido vivo permitió obtener un cuadro más actualizado sobre las características de la lactancia y obvió el problema de la falta de independencia de las observaciones cuando se usan todos los niños de una misma madre.

Aquellas parejas en las cuales la diferencia entre la edad del niño reportada por la madre y la edad calculada a partir de la fecha de nacimiento fue mayor de un año fueron excluidas del estudio porque se consideró que sus datos no eran fiables. No se excluyeron niños muertos ya que todos los fallecidos antes de cumplir un mes de edad fueron lactados en alguna ocasión.

Los factores considerados como potenciales determinantes del inicio de la lactancia al seno fueron clasificados en tres grupos: variables sociodemográficas, características de la atención médica durante el embarazo y el parto y características del embarazo y del niño (cuadro 1). Estos factores fueron definidos siguiendo los cuestionarios modelo del programa mundial de encuestas demográficas y de salud (DHS Modelo A) $(13,16)$.
Las madres fueron clasificadas según el nivel de ingresos del hogar, estimado a partir de las propiedades materiales presentes en el domicilio. A cada tipo de bien le fue asignado un valor relativo de acuerdo con su precio en el mercado. Para cada madre se calculó una puntuación total, sumando estos valores. ${ }^{3}$ Los terciles de la distribución fueron 2,0 y 7,5, y se usaron como puntos de corte para agrupar a las participantes en nivel de ingreso bajo, medio o alto. Esta clasificación estuvo fuertemente asociada con el tipo de residencia (urbana o rural) y con el nivel de educación de la madre (coeficientes Phi iguales a 0,39 y 0,49, respectivamente).

Debido al alto desempleo, a la inestabilidad laboral y a la diversidad de fuentes de ingreso, la determinación del nivel de ingresos a partir del salario o los ingresos mensuales sería poco confiable en esta población. La definición usada permite la combinación de ingresos de distintas fuentes por períodos de tiempo prolongados y una mejor clasificación de las madres según categorías del nivel de ingresos.

Como medida de frecuencia relativa del inicio de la lactancia materna se usó la proporción de niños nunca lactados. Como en la muestra original cada mujer elegible no tenía la misma probabilidad de ser incluida en el estudio (13), para obtener estimaciones representativas de la población la proporción de niños nunca lactados fue ponderada multiplicando cada observación por el inverso de la probabilidad de selección de la madre a la cual correspondía, y acumulando los productos resultantes.

Como medida de asociación para cuantificar el efecto de cada variable sobre el inicio del amamantamiento se utilizó la razón de posibilidades $(\psi)$ (odds ratio, en inglés) (17) y su intervalo de confianza de $95 \%$ (IC95\%), calculado por el método de Cornfield (18).

\footnotetext{
3 Los valores asignados fueron: $1=$ abanico, radio o plancha; 1,5 = estufa de gas; 2 = aparato de música, aire acondicionado, televisión o nevera; 3 = lavadora o motocicleta; $6=$ automóvil.
}

CUADRO 1. Determinantes potenciales del inicio de la lactancia considerados en el presente estudio. República Dominicana, 1991

a) Variables sociodemográficas:

Edad de la madre (años cumplidos) al momento de nacer el niño

Nivel de escolaridad de la madre (último curso completado)

Zona de residencia (urbana o rural)

Nivel socioeconómico (según bienes presentes en el hogar)

Número de partos

Año de nacimiento del niño

Situación laboral de la madre cuando nació el niño

b) Características de la atención médica durante el embarazo y el parto:

Número de exámenes prenatales

Personal de salud a cargo de los exámenes prenatales

Personal de salud a cargo de la atención del parto

Institución en la cual se realizó el parto (hogar, centro público, centro privado)

c) Características del embarazo y del niño:

Enfermedades durante el embarazo declaradas por la madre ${ }^{a}$

Tipo de parto (cesárea o vaginal)

Sexo del niño

Peso del niño al nacer

amenaza de aborto, hemorragia vaginal, placenta previa, hipertensión arterial, cardiopatía, diabetes, tuberculosis 0 enfermedad renal, hepática o tumoral.

Para evaluar el efecto ajustado de cada variable sobre el inicio de la lactancia al seno se usó la regresión logística múltiple (19). La construcción del modelo de regresión tuvo por objetivo encontrar la ecuación más simple que pudiera describir válidamente los datos observados y se hizo siguiendo los criterios de Greenland (20). La eficacia del modelo para describir los datos se evaluó por medio de la prueba de bondad del ajuste (deviance chi square) y por análisis de los residuos (19). Los factores de ponderación no fueron usados durante el análisis multifactorial, ya que no se intentaba obtener promedios nacionales.

\section{RESULTADOS}

En la ENDESA-91 se recolectó información sobre lactancia en 4699 niños. 
De estos, 504 fueron excluidos del presente estudio por ser mayores de 5 años y otros 95 porque sus datos de edad no eran confiables. De los restantes se seleccionó solo al último nacido vivo de cada madre, lo cual redujo la muestra estudiada a 2714 parejas madre-niño.

La muestra estudiada estuvo conformada mayormente por madres menores de 30 años y con 3 hijos o menos (cuadro 2). Apenas 9\% de las madres no habían recibido ninguna instrucción escolar y $55 \%$ solo habían alcanzado el nivel primario. Un poco más de $60 \%$ de los niños era menor de 2 años y una proporción similar procedía de zonas urbanas. Una tercera parte de las madres manifestó haber tenido un tra- bajo asalariado cuando nació su último niño. Los porcentajes ponderados fueron similares a los no ponderados.

De los 2714 niños incluidos en el estudio, 187 (6,9\%) nunca fueron lactados (IC $95 \%$ : 5,7 a 7,9\%). La mayoría de las madres que no iniciaron la lactancia lo hicieron porque el niño rechazó el seno $(33,9 \%)$ o porque no tenían leche suficiente $(30,0 \%)$. El riesgo no ajustado de no lactar fue mínimo en madres de 25 a 29 años y máximo en madres añosas y muy jóvenes (cuadro 3). Además, aumentó con el nivel de escolaridad, con el nivel socioeconómico y en residentes de la zona urbana. Por el contrario, el riesgo disminuyó con el número de partos de la madre y no cambió con la situación

CUADRO 2. Distribución absoluta y porcentual de la población estudiada, según sus características sociodemográficas. República Dominicana, 1991

\begin{tabular}{|c|c|c|c|c|}
\hline $\begin{array}{l}\text { Característica } \\
\text { sociodemográfica }\end{array}$ & $N^{\mathrm{a}}$ & $\%$ & $\begin{array}{c}\% \\
\text { acumulado }\end{array}$ & $\begin{array}{c}\% \\
\text { ponderado }^{b}\end{array}$ \\
\hline \multicolumn{5}{|l|}{ Edad de la madre } \\
\hline$\leq 19$ & 424 & 15,6 & 15,6 & 13,6 \\
\hline 20 a 24 & 925 & 34,1 & 49,7 & 32,9 \\
\hline 25 a 29 & 714 & 26,3 & 76,0 & 27,9 \\
\hline 30 a 34 & 418 & 15,4 & 91,4 & 17,1 \\
\hline 35 a 39 & 173 & 6,4 & 97,8 & 6,4 \\
\hline 40 a 49 & 59 & 2,2 & 100,0 & 1,9 \\
\hline \multicolumn{5}{|l|}{ Número de partos } \\
\hline 1 & 738 & 27,2 & 27,2 & 28,6 \\
\hline 2 & 652 & 24,0 & 51,2 & 23,1 \\
\hline 3 & 564 & 20,8 & 72,0 & 22,2 \\
\hline 4 & 295 & 10,9 & 82,9 & 10,4 \\
\hline$\geq 5$ & 465 & 17,1 & 100,0 & 15,8 \\
\hline \multicolumn{5}{|c|}{ Nivel de escolaridad } \\
\hline Ninguno & 244 & 9,0 & 9,0 & 8,0 \\
\hline Primario & 1493 & 55,0 & 64,0 & 52,0 \\
\hline Secundario & 705 & 26,0 & 90,0 & 28,0 \\
\hline Universitario & 272 & 10,0 & 100,0 & 12,0 \\
\hline \multicolumn{5}{|c|}{ Edad del niño (años) } \\
\hline 0 & 886 & 32,6 & 32,6 & 31,3 \\
\hline 1 & 778 & 28,7 & 61,3 & 28,6 \\
\hline 2 & 460 & 16,9 & 78,3 & 17,1 \\
\hline 3 & 327 & 12,0 & 90,3 & 12,8 \\
\hline 4 & 263 & 9,7 & 100,0 & 10,1 \\
\hline \multicolumn{5}{|l|}{ Zona de residencia } \\
\hline Urbana & 1632 & 60,1 & 60,1 & 62,1 \\
\hline Rural & 1082 & 39,9 & 100,0 & 100,0 \\
\hline \multicolumn{5}{|l|}{$\begin{array}{l}\text { Situación laboral } \\
\text { de la madre }\end{array}$} \\
\hline Sin trabajo & 1827 & 67,3 & 67,3 & 64,8 \\
\hline Con trabajo & 839 & 32,7 & 100,0 & 35,2 \\
\hline
\end{tabular}

aLas diferencias en las sumas de totales por variable se deben a casos sin información.

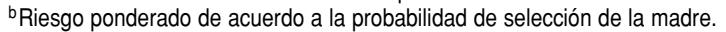

laboral de la madre ni con la edad del niño. Esto último sugiere que durante los últimos 5 años no ha habido cambios importantes en la frecuencia del inicio de la lactancia materna.

En cuanto a la atención del embarazo y el parto, el riesgo de no iniciar la lactancia materna fue independiente del número de exámenes prenatales, pero las madres cuyo examen prenatal estuvo a cargo de una enfermera tuvieron una probabilidad de no iniciar el amamantamiento $46 \%$ mayor que las madres que fueron vistas por médicos (cuadro 4). En comparación con las madres cuyo parto fue atendido por enfermeras, el riesgo de no iniciar la lactancia materna fue más de doble en las atendidas por médicos generales y tres veces y media mayor en las atendidas por obstetras. El riesgo de no iniciar la lactancia materna también aumentó en las madres que tuvieron sus partos en instituciones privadas $(9,8 \%)$ y en instituciones públicas $(5,8 \%)$ en comparación con madres que tuvieron sus partos en el hogar $(2,9 \%)$.

Las madres que padecieron alguna enfermedad importante durante el embarazo (cuadro 1), las que sufrieron cesárea y las que dieron a luz niños con bajo peso al nacer (BPN) tuvieron un riesgo de no iniciar la lactancia al seno considerablemente mayor que las madres con embarazo aparentemente normal (cuadro 5). Por otra parte, el riesgo de no iniciar el amamantamiento fue independiente del sexo del niño.

Los resultados del modelo final de regresión logística reflejaron que de todas las variables estudiadas, las únicas con efecto independiente en el riesgo de no iniciar la lactancia materna fueron el BPN, la presencia de alguna enfermedad durante el embarazo, el número de partos previos y el nivel de ingresos (cuadro 6).

Cuando se incluyeron todas las variables que influyeron significativamente sobre el inicio de la lactancia materna, el BPN fue el factor más fuertemente asociado con el riesgo de no inicio de la lactancia (cuadro 6). Los niños con BPN fueron privados de la lactancia materna casi tres veces más a menudo que los niños de peso adecuado. Entre las madres de niños con 
CUADRO 3. Riesgo absoluto y razón relativa (odds ratio, $\psi$, con su respectivo intervalo de confianza del $95 \%$, IC95\%) de no iniciar la lactancia materna, según características sociodemográficas de la madre. República Dominicana, 1991

\begin{tabular}{|c|c|c|c|c|c|}
\hline \multirow[b]{2}{*}{ Característica sociodemográfica } & \multirow[b]{2}{*}{ Total $^{\mathrm{a}}$} & \multicolumn{2}{|c|}{$\begin{array}{c}\text { Casos que no } \\
\text { inician la lactancia }\end{array}$} & \multirow{2}{*}{$\begin{array}{c}\psi \\
\text { (sin ajustar) }\end{array}$} & \multirow{2}{*}{$\begin{array}{c}\text { IC95\% para } \\
\psi\end{array}$} \\
\hline & & $n$ & $\%$ & & \\
\hline \multicolumn{6}{|l|}{ Edad de la madre } \\
\hline$\leq 19$ & 424 & 35 & 8,2 & 1,0 & - \\
\hline 20 a 24 & 925 & 73 & 7,9 & 0,9 & 0,6 a 1,5 \\
\hline 25 a 29 & 714 & 34 & 4,8 & 0,6 & 0,3 a 0,9 \\
\hline 30 a 34 & 418 & 27 & 6,5 & 0,8 & 0,4 a 1,3 \\
\hline 35 a 39 & 173 & 12 & 6,9 & 0,8 & 0,4 a 1,7 \\
\hline 40 a 49 & 59 & 5 & 8,5 & 1,0 & 0,3 a 2,9 \\
\hline \multicolumn{6}{|l|}{ Nivel de escolaridad } \\
\hline Ninguno/primaria & 1737 & 100 & 5,8 & 1,0 & - \\
\hline Secundaria & 705 & 55 & 7,8 & 1,4 & 1,0 a 2,0 \\
\hline Universitaria & 272 & 31 & 11,4 & 2,1 & 1,3 a 3,3 \\
\hline \multicolumn{6}{|l|}{ Nivel socioeconómico } \\
\hline Bajo & 987 & 44 & 4,5 & 1,0 & - \\
\hline Medio & 925 & 64 & 6,9 & 1,6 & 1,0 a 2,4 \\
\hline Alto & 793 & 78 & 9,8 & 2,3 & 1,6 a 3,5 \\
\hline \multicolumn{6}{|l|}{ Zona de residencia } \\
\hline Rural & 1082 & 56 & 5,2 & 1,0 & - \\
\hline Urbana & 1632 & 130 & 8,0 & 1,6 & 1,1 a 2,2 \\
\hline \multicolumn{6}{|l|}{ No. de partos } \\
\hline 1 & 738 & 77 & 10,4 & 2,7 & 1,6 a 4,7 \\
\hline 2 & 652 & 40 & 6,1 & 1,5 & 0,8 a 2,8 \\
\hline 3 & 564 & 36 & 6,4 & 1,6 & 0,9 a 2,9 \\
\hline 4 & 295 & 14 & 4,7 & 1,2 & 0,5 a 2,5 \\
\hline$\geq 5$ & 465 & 19 & 4,1 & 1,0 & - \\
\hline \multicolumn{6}{|l|}{ Situación laboral } \\
\hline Sin trabajo & 1827 & 128 & 7,0 & 1,0 & - \\
\hline Con trabajo & 839 & 57 & 6,8 & 1,0 & 0,7 a 1,3 \\
\hline \multicolumn{6}{|l|}{ Edad del niño (años) } \\
\hline 0 & 886 & 58 & 6,6 & 1,0 & 0,6 a 1,8 \\
\hline 1 & 778 & 50 & 6,4 & 1,0 & 0,5 a 1,8 \\
\hline 2 & 460 & 32 & 7,0 & 1,1 & 0,6 a 2,1 \\
\hline 3 & 327 & 29 & 8,9 & 1,4 & 0,7 a 2,7 \\
\hline 4 & 263 & 17 & 6,5 & 1,0 & - \\
\hline
\end{tabular}

aLas diferencias en las sumas de totales por variable se deben a casos sin información.

BPN que nunca lactaron al seno la proporción de las que dijeron que la causa de no haberlo hecho fue enfermedad del niño fue cinco veces mayor que en las madres de niños de peso adecuado que tampoco amamantaron $(45,8 \mathrm{y}$ $8,1 \%$, respectivamente).

Las madres con enfermedades de importancia durante el embarazo tuvieron un riesgo de no iniciar la lactancia al seno más de dos veces mayor que el de las madres sin enfermedades manifiestas. Este resultado no cambió cuando se excluyeron del análisis 81 madres ( $3 \%$ de la muestra) que no tuvieron examen prenatal. Además, el riesgo de no iniciar el amamantamiento fue en madres primerizas casi el doble que en multíparas.

Por último, el nivel de ingresos estuvo fuertemente asociado con la probabilidad de no iniciar la lactancia materna. En comparación con las madres de bajo nivel de ingresos, las madres de nivel medio o alto tuvieron un riesgo de no iniciar el amamantamiento 59 y $111 \%$ mayor, respectivamente (cuadro 6).

Cuando se tuvo en cuenta el nivel de ingresos desapareció el efecto de las características de la atención médica sobre el riesgo de no iniciar la lactancia materna. De manera similar, una vez considerada la presencia de enfermedad durante el embarazo, el BPN y el número total de partos, el tipo de personal que llevó a cabo los exámenes prenatales no influyó en el riesgo de no iniciar la lactancia.

Según los resultados de la prueba de ajuste y el análisis de los residuos (19), el modelo de regresión obtenido describe apropiadamente los datos observados ( $\chi^{2}$ de ajuste del modelo, 24,0 , con $17 \mathrm{gl} ; P=0,12)$ y los resultados no se deben a la presencia de observaciones con influencia excesiva en el modelo final.

\section{DISCUSIÓN}

Algunas características del estudio pueden haber sesgado sus resultados. Dado que solo $78 \%$ de todas las mujeres seleccionadas participó en la encuesta original (13), podría haber algún tipo de sesgo de selección ya que el inicio y duración de la lactancia podrían estar asociados con la participación en el estudio. Lamentablemente, no existe información que permita evaluar la dirección y magnitud de tal sesgo potencial (13). Por otra parte, el sesgo resultante del uso de una muestra de supervivientes en el presente estudio debe ser mínimo, ya que la mortalidad en mujeres en edad fértil es un evento raro.

Otra posible fuente de error fue la ausencia de datos sobre potenciales factores de confusión como la conducta de alojamiento conjunto de la madre con el recién nacido (21), la preferencia del padre (22), la educación sobre la lactancia durante el embarazo y el parto (23), y la obesidad (24). Sin embargo, es razonable esperar que el efecto de confusión de esas variables haya sido parcialmente controlado al incluir el nivel de ingresos y el lugar del parto en el análisis multifactorial.

Por último, a pesar de que la calidad de los datos procedentes de las encuestas demográficas y de salud ha sido comprobada en distintos estudios (25, 26), la validez de la notificación 
materna de enfermedades durante el embarazo no parece haber sido evaluada.

En el presente estudio $93 \%$ de las madres reportaron haber iniciado la lactancia materna. A pesar de proceder de una muestra diferente, esta cifra solo fue ligeramente inferior a la reportada por Ogando et al. (27) en un estudio de cohorte en una muestra de madres de varias ciudades que tuvieron sus partos en hospitales públicos.

En zonas rurales la frecuencia del inicio de la lactancia al seno fue ligeramente mayor (95\%) que en zonas urbanas $(92 \%)$. En otros países en desarrollo se ha notificado un nivel alto de inicio de la lactancia materna en zonas urbanas (28) y a menudo se han registrado niveles aun más altos en zonas rurales (29).

Nuestros resultados, como los de Ogando et al. (27), sugieren que en esta población la frecuencia del inicio del amamantamiento se ha mantenido estable durante los últimos cinco años, lo que va en contra de la afirmación de Velzeboer et al. (15), quienes basándose en una revisión no formal de fuentes secundarias han señalado que en los últimos años hubo una disminución en el inicio de la lactancia materna entre los pobres de las zonas urbanas y un aumento en las clases media y alta.

La presencia de enfermedades durante el embarazo, el BPN, el número de partos y el nivel socioeconómico fueron las únicas variables independientemente asociadas al riesgo de no iniciar la lactancia al seno. Aunque podría ser consecuencia de errores de información, el aumento del riesgo de no iniciar la lactancia materna en madres con enfermedades durante el embarazo coincide con lo reportado por Mata et al. (30) en madres costarricenses. Este aumento del riesgo podría deberse a que las madres con enfermedades durante el embarazo, por su propia enfermedad o por problemas del recién nacido, son más frecuentemente separadas de sus niños durante el período perinatal, lo cual dificulta el inicio de la lactancia (31). También es posible que algunas enfermedades relacionadas con el

CUADRO 4. Riesgo absoluto y razón relativa (odds ratio, $\psi$, con su respectivo intervalo de confianza del $95 \%$, IC95\%) de no iniciar la lactancia materna, según características de la atención médica durante el embarazo. República Dominicana, 1991

\begin{tabular}{|c|c|c|c|c|c|}
\hline \multirow[b]{2}{*}{ Característica de la atención médica } & \multirow[b]{2}{*}{ Total $^{a}$} & \multicolumn{2}{|c|}{$\begin{array}{c}\text { Casos que no } \\
\text { inician la lactancia }\end{array}$} & \multirow{2}{*}{$\begin{array}{c}\psi \\
\text { (sin ajustar) }\end{array}$} & \multirow{2}{*}{$\begin{array}{c}\text { IC95\% para } \\
\psi\end{array}$} \\
\hline & & $n$ & $\%$ & & \\
\hline \multicolumn{6}{|l|}{ No. de exámenes prenatales } \\
\hline 0 a 4 & 669 & 43 & 6,4 & 1,0 & - \\
\hline 5 a 7 & 874 & 54 & 6,2 & 1,0 & 0,6 a 1,4 \\
\hline 8 a 9 & 674 & 55 & 8,2 & 1,3 & 0,9 a 1,9 \\
\hline$\geq 10$ & 485 & 32 & 6,6 & 1,0 & 0,7 a 1,6 \\
\hline \multicolumn{6}{|l|}{ Exámenes prenatales } \\
\hline Por médico & 1360 & 77 & 5,7 & 1,0 & - \\
\hline Por enfermera & 1256 & 104 & 8,3 & 1,5 & 1,1 a 1,9 \\
\hline Otro & 97 & 4 & 4,1 & 0,7 & 0,3 a 1,9 \\
\hline \multicolumn{6}{|l|}{ Atención durante el parto } \\
\hline Por enfermera & 201 & 5 & 2,5 & 1,0 & - \\
\hline Por médico gral. & 1056 & 58 & 5,5 & 2,2 & 0,9 a 5,4 \\
\hline Por obstetra & 1300 & 112 & 8,6 & 3,5 & 1,4 a 8,4 \\
\hline \multicolumn{6}{|l|}{ Lugar del parto } \\
\hline Hogar/otro & 205 & 6 & 2,9 & 1,0 & - \\
\hline Instit. pública & 1666 & 97 & 5,8 & 2,0 & 0,9 a 4,5 \\
\hline Instit. privada & 843 & 83 & 9,8 & 3,4 & 1,5 a 7,6 \\
\hline
\end{tabular}

aLas diferencias en las sumas de totales por variable se deben a casos sin información.

CUADRO 5. Riesgo absoluto y razón relativa (odds ratio, $\psi$, con su respectivo intervalo de confianza del $95 \%$, IC $95 \%$ ) de no iniciar la lactancia materna, según características del embarazo y del niño. República Dominicana, 1991

\begin{tabular}{|c|c|c|c|c|c|}
\hline \multirow[b]{2}{*}{ Característica del embarazo y del niño } & \multirow[b]{2}{*}{ Total $^{\mathrm{a}}$} & \multicolumn{2}{|c|}{$\begin{array}{c}\text { Casos que no } \\
\text { inician la lactancia }\end{array}$} & \multirow{2}{*}{$\begin{array}{c}\psi \\
\text { (sin ajustar) }\end{array}$} & \multirow{2}{*}{$\begin{array}{c}\text { IC95\% para } \\
\psi\end{array}$} \\
\hline & & $n$ & $\%$ & & \\
\hline \multicolumn{6}{|l|}{$\begin{array}{l}\text { Enfermedad durante } \\
\text { el embarazo }\end{array}$} \\
\hline No & 2320 & 134 & 5,8 & 1,0 & - \\
\hline Sí & 392 & 52 & 13,3 & 2,3 & 1,7 a 3,1 \\
\hline \multicolumn{6}{|l|}{ Tipo de parto } \\
\hline Natural & 2098 & 129 & 6,2 & 1,0 & - \\
\hline Cesárea & 606 & 55 & 9,1 & 1,5 & 1,1 a 2,0 \\
\hline \multicolumn{6}{|l|}{ Peso al nacer } \\
\hline$\geq 2500 \mathrm{~g}$ & 2320 & 134 & 5,8 & 1,0 & - \\
\hline$<2500 \mathrm{~g}$ & 231 & 35 & 15,2 & 2,6 & 1,8 a 3,7 \\
\hline \multicolumn{6}{|l|}{ Sexo del neonato } \\
\hline$q$ & 1312 & 83 & 6,3 & 1,0 & - \\
\hline$\hat{0}$ & 1402 & 103 & 7,4 & 1,2 & 0,9 a 1,5 \\
\hline
\end{tabular}

a Las diferencias en las sumas de totales se deben a casos sin datos.

Los resultados no cambian de forma significativa si se excluyen 81 madres que no tuvieron visitas prenatales.

embarazo estén asociadas por medio de mecanismos hormonales con una disminución en la producción de leche materna que obstaculice el inicio de la lactancia.
En los niños con BPN no tuvo lugar el inicio de la lactancia al seno casi tres veces más frecuentemente que en los niños de peso adecuado. Hallazgos similares han sido reportados en otros 
CUADRO 6. Razón relativa (odds ratio, $\psi$, con su respectivo intervalo de confianza del $95 \%$, IC95\%) de no iniciar la lactancia materna, ajustada según características sociodemográficas, de la atención médica y del niño. República Dominicana, 1991

\begin{tabular}{lccc}
\hline \multicolumn{1}{c}{ Característica } & $\psi$ & IC95\% & $P$ \\
\hline Enfermedad durante el embarazo & 1,0 & - & - \\
$\quad$ No & 2,3 & 1,6 a 3,2 & $<0,001$ \\
Sí & 1,0 & - & - \\
Bajo peso al nacer & 2,9 & 1,9 a 4,4 & $<0,001$ \\
$\quad$ No & 1,0 & & - \\
Sía & 1,9 & 1,4 a 2,7 & - \\
No. de partos & & & 0,001 \\
Multípara & 1,0 & - & $<, 023$ \\
Primeriza & 1,7 & 1,1 a 2,4 & $<, 001$ \\
Nivel socioeconómico & 2,1 & 1,4 a 3,1 & \\
$\quad$ Bajo & Medio & & \\
Alto & & &
\end{tabular}

ancluye tres niños sin dato de peso considerados por su madre como muy pequeños al nacer.

estudios (32, 33). Winikoff et al. (28) han reportado un mayor riesgo de no iniciar la lactancia materna en madres cuyos niños tienen problemas en el período perinatal inmediato. La disminución en el inicio del amamantamiento en niños con BPN podría estar mediada por la separación madre-niño durante los primeros días de vida del recién nacido (31), así como por la incapacidad del niño con BPN para succionar con la fuerza suficiente para estimular la producción de leche materna. A favor de este argumento va el que, en comparación con las madres de niños no lactados de peso adecuado, las madres de niños no lactados con BPN manifestaron con mucha mayor frecuencia que la enfermedad del niño era la causa de no darle el pecho.

En el presente estudio, el riesgo de no iniciar el amamantamiento fue casi el doble en las madres primerizas que en las multíparas. Los resultados de un estudio realizado en Puerto Rico (34) también indican un mayor riesgo de no iniciar la lactancia al seno en mujeres primíparas. Sin embargo, en otros estudios (28) no se ha encontrado asociación entre el número de partos previos y el inicio de la lactancia materna, probablemente debido a la alta frecuencia de esta última. La menor frecuencia del inicio de la lactancia al seno en madres primerizas podría estar asociada a falta de conocimiento de sus beneficios.

El incremento en el riesgo de no iniciar la lactancia materna a medida que aumenta el nivel de ingresos ha sido también notificado en otros estudios $(28,35,36)$, a pesar de que se han usado distintas definiciones de esta variable. La interpretación de este hallazgo es compleja ya que el nivel de ingresos es una variable asociada a otros factores más difíciles de medir, como valores y creencias socioculturales (37).

Distintos autores $(28,30,34)$ coinciden en señalar los efectos adversos de la "industrialización" y la "occidentalización de las formas de vida" en el inicio y duración de la lactancia materna. Estos efectos parecen ser mediados por la introducción y mercadeo agresivo de sustitutos de la leche materna, la introducción de nuevas tecnologías y prácticas en las salas de maternidad y atención perinatal y el cambio de actitudes y creencias en torno a la lactancia materna. El impacto podría ser mayor en grupos sociales que cuentan con mayor poder de consumo y mayor acceso a la atención médica y a la educación occidentalizada. Los cambios en el inicio de la lactancia materna experimentados por la población de Puerto Rico de 1946 a 1982 (34) son un buen ejemplo de estos efectos de la occidentalización.

La falta de asociación entre la situación laboral de la madre y el inicio de la lactancia materna observada en este estudio, ha sido reportada previamente por Gómez et. al. (14) y por Johnson (38) en mujeres dominicanas y por otros autores en otros países en desarrollo (28). Este hallazgo no es sorprendente ya que la ley concede a las mujeres dominicanas 3 meses de licencia remunerada posparto. Además, solo $31 \%$ de las madres en este estudio tenían un trabajo asalariado y de estas la mitad llevaba habitualmente consigo el niño al trabajo.

La edad de la madre no influyó en el inicio de la lactancia al seno. En tres de cuatro estudios en países en desarrollo (28) tampoco se encontró asociación alguna entre la edad de la madre y el riesgo de no iniciar el amamantamiento, y en el único estudio en el que se detectó una asociación significativa (31) la frecuencia de inicio de la lactancia materna disminuyó con la edad de la madre.

La falta de un efecto de la educación y de las características de atención médica en el presente estudio podría también explicarse por la inclusión del nivel de ingresos y de las enfermedades durante el embarazo en el modelo de regresión.

Aunque el inicio de la lactancia materna fue muy frecuente $y$ no parece haber disminuido durante los últimos años, en otros países se ha observado una tendencia a la disminución $(11,34)$ asociada con el crecimiento económico. En consecuencia, es prudente desarrollar acciones tendentes a prevenir un deterioro en la frecuencia de inicio de la lactancia en las mujeres dominicanas.

En una revisión de estudios de evaluación de programas de promoción de la lactancia, Tognetti (29) ha señalado que en la mayoría de estos programas no se ha prestado atención a los factores que podrían ayudar a definir los grupos a los que se dirige fundamentalmente el programa, a pesar 
de que este es un aspecto fundamental para el éxito de la intervención.

Actualmente el Programa Nacional de la Lactancia Materna (15) en la República Dominicana está dirigido a las usuarias del sistema público de salud. Aunque es posible argumentar que los efectos de no lactar podrían ser peores en ese grupo, de acuerdo a los resultados del presente estudio, las primerizas o las que han tenido pocos partos, las que tienen enfermedades importantes durante el embarazo, las que tienen niños con BPN y las de nivel de ingresos alto deberían ser grupos a los que se dirigieran específicamente las acciones de promoción del inicio de la lactancia.

\section{REFERENCIAS}

1. Feachem RG, Koblinski MA. Interventions for the control of diarrhoeal diseases among young children: promotion of breast feeding. Bull World Health Organ 1984;62:271-291.

2. Mahalanabis D, Alam AN, Rahman N, Hasnat A. Prognostic indicators and risk factors for increased duration of acute diarrhoea and for persistent diarrhoea in children. Int J Epidemiol 1991;20:1064-1072.

3. Forman MR, Graubard BI, Hoffman HJ, Beren R, Harley EE, Bennet P. The Pima infant feeding study: Breast-feeding and respiratory infections during the first year of life. Int $J$ Epidemiol 1984;13:447-453.

4. Victoria CG, Smith PG, Vaughan JP, et al. Evidence for protection by breast-feeding against infant deaths from infectious diseases in Brazil. Lancet 1987;2:319-322..

5. Butte NF, Garza C, Smith EO, Nichols BL. Human milk intake and growth in exclusively breast-fed infants. J Pediatr 1984;104:187-195.

6. Short RV, Lewis PR, Renfree MB, Shaw G. Contraceptive effects of extended lactational amenorrhoea: beyond the Bellagio Consensus. Lancet 1991;337:715-717.

7. Huffman SL. Determinants of breastfeeding in developing countries: overview and policy implications. Stud Fam Plann 1984;15:170-183.

8. Pérez Escamilla R. Breastfeeding in Africa and the Latin American and Caribbean region: the potential role of urbanization. J Trop Pediatr 1994;40:137-143.

9. Forman MR. Review of research on the factors associated with choice and duration of infant feeding in less-developed countries. Pediatrics 1984;74(4 pt 2):667-694.

10. Winikoff B, Laukaran VH. Breast feeding and bottle feeding controversies in the developing world: evidence from a study in four countries. Soc Sci Med 1989;29:859-868.

11. Division of Family Health WH. The prevalence and duration of breastfeeding: critical review of available information. World Health Stat Q 1982;35:92-112.

12. Secretaría de Estado de Salud Pública y Asistencia Social, Consejo Nacional de Población y Familia, Institute for Resource Development/Westinghouse. Encuesta demográfica y de salud 1986 (DHS-86). Santo Domingo: CONAPOFA; 1987:21-24.

13. Instituto de Estudios de Población y Desarrollo, Oficina Nacional de Planificación, IRD/Macro International Inc. Encuesta demográfica y de salud 1991. Santo Domingo: PROFAMILIA; 1992:131-146.

14. Gómez E, Montero R, Canario M. Patrones de lactancia materna en el Distrito Nacional de la
República Dominicana. En: CENISMI. Estado actual de la lactancia materna en la República Dominicana. Santo Domingo: CENISMI; 1987:5-15.

15. Velzeboer M, Coen J, Alas de Chavez A, Fischer M. El estado de la lactancia materna en la República Dominicana: prácticas y promoción. Washington, DC: United States Agency for International Development, Mother Care, LAC Health and Nutrition Sustainability; 1992:1-112.

16. Sullivan JM. The collection of mortality data in WFS and DHS surveys. En: Vallin J, D'Souza S, Palloni A. Measurement and analysis of mortality: new approaches. Oxford: Clarendon Press; 1990:48-63.

17. Bautista LE. "Razón relativa" y "tasa relativa" como traducciones de odds ratio y hazard ratio (carta). Bol Oficina Sanit Panam 1995;119: 278-279.

18. Cornfield J. A statistical problem arising from retrospective studies. En: Neyman J. Proceedings of the Third Berkeley Symposium. Berkeley: University of California Press; 1956:135-148.

19. Hosmer DW, Lemeshow S. Applied logistic regression. New York: John Wiley; 1989.

20. Greenland S. Modeling and variable selection in epidemiologic analysis. Am J Public Health 1989;79:340-349.

21. Pérez Escamilla R, Segura Millán S, Pollitt E, Dewey KG. Effect of the maternity ward system on the lactation success of low-income urban Mexican women. Early Hum Dev 1992;31:25-40.

22. Jones DA. The choice to breast feed or bottle feed and influences upon that choice: a survey of 1525 mothers. Child Care Health Dev 1987:13:75-85.

23. Winikoff B, Castle MA. The influence of health services on infant feeding. En: Winikoff B, Castle MA, Laukaran VH. Feeding infants in four societies: causes and consequences of mothers's choices. New York: Greenwood Press; 1988:147-164

24. Rutishauser IH, Carlin JB. Body mass index and duration of breast feeding: a survival analysis during the first six months of life. J Epidemiol Community Health 1992;46:559-565.

25. Goldman N, Rutstein SO, Singh S. Assesment of the quality of data in 41 WFS surveys: a comparative approach. Voorburg, Netherlands: International Statistics Institute; 1985:5-83.

26. Eaton Evans J, Dugdale AE. Recall by mothers of the birth weights and feeding of their children. Hum Nutr Appl Nutr 1986;40:171-175.

27. Ogando E, Romero H, Soriano G. Monitoreo del efecto de las políticas económicas y sociales en el bienestar infantil 1988-1991. Santo Domingo: Centro Nacional de Investigación en Salud Materno-Infantil, Oficina Nacional de Planificación, UNICEF; 1992:136.

28. Winikoff B. Summary. En: Winikoff B, Castle MA, Laukaran VH. Feeding infants in four societies: causes and consequences of mothers' choices. New York: Greenwood Press; 1988:227-246.

29. Tognetti J. Evaluating breastfeeding promotion programmes. En: Jelliffe DB, Jelliffe EFP. Programmes to promote breastfeeding. New York: Oxford University Press; 1988:405-419.

30. Mata L, Sáenz P, Araya JR, Allen MA, García ME, Rodríguez ME, Carvajal JJ. Promotion of breastfeeding in Costa Rica: the Puriscal study. En: Jelliffe DB, Jelliffe EFP. Programmes to promote breastfeeding. New York: Oxford University Press; 1988:55-69.

31. Winikoff B, Durongdej S, Cerf BJ. Infant feeding in Bangkok, Thailand. En: Winikoff B, Castle MA, Laukaran VH. Feeding infants in four societies: causes and consequences of mothers choices. New York: Greenwood Press; 1988: $15-41$.

32. Xavier CC, Jorge SM, Gonçalves AL. Prevalencia do aleitamento materno em recemnascidos de baixo peso. Rev Saude Publica 1991;25:381-387.

33. Ever-Hadani P, Seidman DS, Manor O, Harlap S. Breast feeding in Israel: maternal factors associated with choice and duration. J Epidemiol Community Health 1994; 48:281-285.

34. Becerra JE, Smith JC. Breastfeeding patterns in Puerto Rico. Am J Public Health 1990;80: 694-697.

35. Manderson L. 'These are modern times': infant feeding practice in Peninsular Malaysia. Soc Sci Med 1984;18:47-57.

36. Grossman LK, Fitzsimmons SM, Larsen Alexander JB, Sachs L, Harter C. The infant feeding decision in low and upper income women. Clin Pediatr (Phila) 1990;29:30-37.

37. Liberatos P, Link BG, Kelsey JL. The measurement of social class in epidemiology. Epidemiologic Reviews 1988;10:87-121.

38. Johnson C. Nutritional status in the Dominican Republic. Santo Domingo: Tuft University School of Nutrition/USAID; 1987.

Manuscrito recibido el 22 de mayo de 1995 y aceptado para publicación en version revisada el 22 de febrero de 1996. 
ABSTRACT The determinants of initiating breast-feeding vary among different populations, but knowledge of them is of fundamental importance for guiding programs to promote breast-feeding. Data from the Demographic and Health Survey of 1991 in the Dominican Republic were used to identify factors associated with the initiation of breastfeeding in a random sample of women of reproductive age. Approximately $93 \%$ of 2714 mothers reported having begun to breast-feed their last live-born child who was currently under 5 years of age, and that percentage had not changed substantially in the past 5 years. A logistic regression analysis was done in which odds ratios $(\psi)$ were calculated as measures of association. Women who had suffered some type of illness during pregnancy $(\psi=2.3)$, those whose child had a low birthweight $(\psi=2.9)$, primiparas $(\psi=1.9)$, and those with medium $(\psi=1.6)$ or high $(\psi=2.1)$ income levels showed a significantly higher risk of not starting to breast-feed. These women should

\section{Factors associated with the initiatio reast-feec Dominican Republic}

be considered priority groups by breast-feeding promotion programs.

\begin{tabular}{|c|c|}
\hline \multicolumn{2}{|c|}{ V Congreso Latinoamericano de Salud Ocupacional (ALS0 ’97) } \\
\hline $\begin{array}{l}\text { Fechas: } \\
\text { Lugar: } \\
\text { Tema: } \\
\text { La Asociación Latinoa } \\
\text { organizado por la Sociedad } \\
\text { principal se tratarán otras ma } \\
\text { su aplicación, estrés, toxicolc } \\
\text { empresas, manejo de riesgos } \\
\text { ALSO invita a todos lo } \\
\text { tección de la salud en el traba } \\
\text { de la calidad laboral, y repre } \\
\text { actividad científica. En el ma } \\
\text { internacional en la cual diver } \\
\text { ductos y servicios. }\end{array}$ & $\begin{array}{l}19 \text { a } 22 \text { de mayo de } 1997 \\
\text { Quito, Ecuador } \\
\text { La ergonomía en la prevención de los trastornos } \\
\text { causados por movimientos repetitivos } \\
\text { mericana de Salud Ocupacional (ALSO) anuncia este congreso } \\
\text { cuatoriana de Seguridad y Salud Ocupacional. Además del tema } \\
\text { erias relacionadas como el cáncer, los programas informáticos y } \\
\text { gía laboral, seguridad e higiene industrial, servicios médicos de } \\
\text { protección ambiental y otros. } \\
\text { profesionales vinculados con la prevención de riesgos y la pro- } \\
\text { o así como a gerentes de recursos humanos y de mantenimiento } \\
\text { sentantes de sindicatos y comités laborales a participar en esta } \\
\text { co del congreso tendrá lugar también PREVEXPO '97, una feria } \\
\text { as empresas expondrán sus publicaciones, videos, y otros pro- } \\
\text { Información } \\
\text { Secretaría Oficial de ALSO '97 } \\
\text { Cuatoriana de Seguridad y Salud Ocupacional } \\
\text { Casilla } 7015 \\
\text { Teléfono: (593-4) } 330706 \\
\text { Fax: (593-4) } 580189\end{array}$ \\
\hline
\end{tabular}

